\title{
ISOLATED SINGULARITIES OF THE SCHRÖDINGER EQUATION WITH A GOOD POTENTIAL
}

\author{
JUAN LUIS VAZQUEZ AND CECILIA YARUR
}

\begin{abstract}
We study the behaviour near an isolated singularity, say 0 , of nonnegative solutions of the Schrödinger equation $-\Delta u+V u=0$ defined in a punctured ball $0<|x|<R$. We prove that whenever the potential $V$ belongs to the Kato class $K_{n}$ the following alternative, well known in the case of harmonic functions, holds: either $|x|^{n-2} u(x)$ has a positive limit as $|x| \rightarrow 0$ or $u$ is continuous at 0 . In the first case $u$ solves the equation $-\Delta u+V u=a \delta$ in $\{|x|<R\}$. We discuss the optimality of the class $K_{n}$ and extend the result to solutions $u \geq 0$ of $-\Delta u+V u=f$.
\end{abstract}

\section{INTRODUCTION}

In this paper we are concerned with the behaviour around an isolated singularity of the solutions of the equation

$$
L u \equiv-\Delta u+V u=f,
$$

where $V$ is a potential in the Kato class, $f$ belongs to a suitable $L^{p}$ space and the solution is assumed to be not too negative. The equation is posed in a domain $\Omega \subset \mathbf{R}^{n}$ with singularities at some isolated points. Without loss of generality we may assume that $\Omega$ is the punctured ball $B_{R}^{*}=B_{R}-\{0\}$, with $B_{R}=\left\{x \in \mathbf{R}^{n}:|x|<R\right\}$, and we investigate the behaviour as $|x| \rightarrow 0$. We also assume for simplicity that $n \geq 3$, though the results have natural counterparts in two dimensions.

In the simplest case where $V$ and $f$ vanish identically this behaviour is well known. In fact, given any nonnegative harmonic function $u=u(x)$ defined in $B_{R}^{*}$, there exists the limit

$$
\lim _{|x| \rightarrow 0}|x|^{n-2} u(x)=a \geq 0 .
$$

Moreover, if $a>0$ then $u$ exhibits a singularity at 0 and the equation

$$
-\Delta u=c_{n} a \delta
$$

Received by the editors September 23, 1987 and, in revised form, March 21, 1988.

1980 Mathematics Subject Classification (1985 Revision). Primary $35 J 05$.

Key words and phrases. Isolated singularities, Schrödinger equation, Kato potentials.

The first author was partially supported by CAYCIT Grant 2805-83.

The second author was partially supported by P.N.U.D.-UNESCO, FONDECYT 5061, 1986. 
is satisfied in $D^{\prime}\left(B_{R}\right)$. Here $\delta$ denotes the Dirac mass at the origin and $c_{n}=$ $(n-2) s_{n}$, where $s_{n}$ is the $(n-1)$-measure of the unit sphere $S_{n-1}=\{x \in$ $\left.\mathbf{R}^{n}:|x|=1\right\}$. Such a singularity is called a weak singularity. In case $a=0, u$ can be extended to 0 as a harmonic function, i.e. the singularity is removable.

The fact that any isolated singularity is either weak or removable holds true for the nonnegative solutions of a wide class of elliptic equations, cf. [GS, S1, S2, SW]. In [S2] Serrin established the result for the Schrödinger equation

$$
L u \equiv-\Delta u+V u=0
$$

with $V \in L_{\text {loc }}^{q}\left(B_{R}\right), q>n / 2$.

We consider here the same problem for the class of Kato potentials defined as follows. A function $V \in L_{\mathrm{loc}}^{1}\left(B_{R}\right)$ belongs to $K_{n}=K_{n}\left(B_{R}\right)$ if and only if

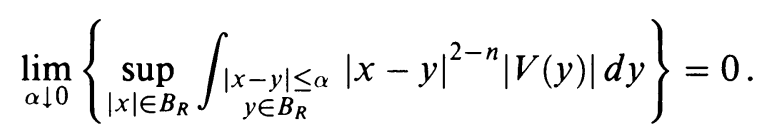

In case we replace $x, y \in B_{R}$ by $x, y \in B_{R^{\prime}}$ with $0<R^{\prime}<R$ we obtain the class $K_{n, \text { loc }}=K_{n \text {,loc }}\left(B_{R}\right)$. We may always pass from $K_{n, \text { loc }}$ to $K_{n}$ by reducing the size of the ball where we work. $K_{n}$ contains the space $L^{q}\left(B_{R}\right)$ if $q>n / 2$, and it also contains other potentials as is easily seen in the radial case. Namely, if $V=V(|x|)$ then $V \in K_{n}$ if

$$
\int_{0}^{R} r|V(r)| d r<\infty,
$$

cf. [AS]. On the other hand $K_{n}$ does not contain $L^{n / 2}\left(B_{R}\right)$, as the following example shows: take $V(y)=|y|^{-2}(\log |y|)^{-\alpha}$ with $1 \geq \alpha>2 / n$.

The class $K_{n}$ has received much attention recently in connection with the Schrödinger equation. Thus, Kato $[\mathrm{K}]$ proved the selfadjointness of the operator $L=-\Delta+V$ when $V \in K_{n}$. Aizenman and Simon [AS] have proved the Harnack inequality for nonnegative solutions of (3), cf. also [CFG]. Simon in his survey [Si] considers $K_{n}$ as the natural class of good potentials for different aspects in the theory of the Schrödinger equation.

Our results show that $K_{n}$ is a class of good potentials also from the point of view of isolated singularities.

Thus, our first result is

Theorem 1. Let $V \in K_{n}\left(B_{R}\right)$ and let $u \in L_{\mathrm{loc}}^{1}\left(B_{R}^{*}\right)$ be such that

(i) $u \geq 0$ in $B_{R}$,

(ii) $\Delta u \in L_{\mathrm{loc}}^{1}\left(B_{R}^{*}\right)$,

(iii) $\Delta u=V \cdot u$ in $B_{R}^{*}$.

Then there exists a constant $\alpha \geq 0$ such that

$$
\begin{gathered}
-\Delta u+V u=\alpha \delta \quad \text { in } D^{\prime}\left(B_{R}\right), \\
\lim _{|x| \rightarrow 0}|x|^{n-2} u(x)=\alpha / c_{n} .
\end{gathered}
$$


Moreover, if $\alpha=0$ then $u$ is a continuous solution of (4) in $B_{R}$, i.e. $u$ satisfies (ii), (iii) in $B_{R}$.

Here $c_{n}$ is the constant appearing in (3). Equalities and inequalities between integrable functions are to be understood a.e. in the corresponding domain. The theorem has as a consequence the following removability result.

A nonnegative solution of (4) in $B_{R}^{*}$ such that either $u(x)=o\left(|x|^{2-n}\right)$ as $|x| \rightarrow 0$ or $u \in L_{\mathrm{loc}}^{p}\left(B_{R}\right)$ for $p=n /(n-2)$ is in fact a continuous solution in $B_{R}$.

In the work [VY2] (cf. also [VY1]) a study is made of the isolated singularities of the equation $L u=0$ when the potentials $V$ are radially symmetric ${ }^{1}$. In particular, Theorem 1 is proved under this restriction. Moreover, it is shown that the class $K_{n}$ is optimal in the following sense. If $V \in C(0, R]$ is nonnegative and $V$ does not satisfy (6) then any nonnegative solution of (4) in $B_{R}^{*}$ which is singular satisfies as $|x| \rightarrow 0$

$$
\lim _{|x| \rightarrow 0}|x|^{n-2} u(x)=+\infty \text {. }
$$

The result actually holds for potentials with changing sign provided that $V^{-}(r)$ $=\max (-V(r), 0) \in K_{n}$, cf. [VY2, Propositions 2.2, 2.3]. Moreover, if for such $V$ and $u$ we can assert that $V u \in L_{\text {loc }}^{1}\left(B_{R}\right)$ then the singularity is automatically removable, cf. [VY2, Theorem B].

Theorem 1 can be extended to solutions of possibly changing sign for equation (1) with $f$ in some $L^{p}$-space by means of recent results in potential theory for Schrödinger operators with $V$ in $K_{n}$, taken from Cranston, Fabes and Zhao [CFZ]. In particular, we use the fact that for $r$ small enough there exists a fundamental solution for $L$ in $B_{r}$, i.e. a function $U \in C\left(B_{r}^{*}\right)$ which satisfies (7) and (8) with $\alpha=1$. Then we obtain

Theorem 2. Let $V \in K_{n}\left(B_{R}\right)$ and let $u \in L_{\text {loc }}^{1}\left(B_{R}^{*}\right)$ be such that

(i) $u \geq O\left(|x|^{2-n}\right)$ as $|x| \rightarrow 0$,

(ii) $u \in L_{\mathrm{loc}}^{1}\left(B_{R}^{*}\right)$ and

(iii) $\Delta u=V u+f$ in $D^{\prime}\left(B_{R}^{*}\right)$ and $f \in L^{p}\left(B_{R}\right)$ with $p>n / 2$.

Then (7) and (8) hold for some $\alpha \in \mathbf{R}$. Moreover, for $r$ small enough we may write

$$
u(x)=\alpha U(x)+g(x) \text { in } B_{r},
$$

where $U$ is the fundamental solution for $L$ in $B_{r}$ and $g$ is a continuous solution of (4) in $B_{r}$.

\section{Proof of Theorem 1}

We begin with some convenient notation and definitions. A solution of $L u \equiv$ $-\Delta u+V u=f$ in a domain $\Omega \subset \mathbf{R}^{n}$ is a function $u \in L_{\text {loc }}^{1}(\Omega)$ such that

\footnotetext{
${ }^{1}$ They are also assumed to be continuous for $0<|x|<R$ but this restriction is inessential for the results.
} 
$V u \in L_{\text {loc }}^{1}(\Omega)$ and the equation holds in the sense of distributions in $\Omega$. In this paper $\Omega$ will be a ball or a punctured ball. We shall often use averages over spheres: if $u \in L_{\mathrm{loc}}^{1}\left(B_{R}^{*}\right)$ we define its angular average, $\bar{u}:(0, R) \rightarrow \mathbf{R}$ by

$$
\bar{u}(r)=s_{n}^{-1} \int_{S_{n-1}} u(r, \sigma) d \sigma,
$$

where $(r, \sigma)$ are spherical coordinates in $B_{R}$. Clearly $\bar{u} \in L_{\mathrm{loc}}^{1}(0, R)$ and $\overline{\Delta u}=\Delta(\bar{u})$.

The proof of Theorem 1 proceeds in three steps. First, we use a sharp version of the continuity results of [AS]. In fact, a careful scrutiny of their proofs of the Harnack inequality plus a scaling argument gives

Lemma 3. Let $u$ be a nonnegative solution of $L u=0$ in $B_{R}^{*}$ with $V \in K_{n}$. Then there exists a constant $c$, depending only on $n$ and the local norms of $V$ in $K_{n}$, such that for $0<r<R / 2$

$$
\max _{|x|=r} u(x) \leq c \min _{|x|=r} u(x) .
$$

Proof. Let us prove first that there exists $a \in(0,1)$ such that for every $x_{0}$, $0<\left|x_{0}\right|<R / 2$, we have

$$
\sup \left\{u(x): x \in B_{a \rho}\left(x_{0}\right)\right\} \leq C \inf \left\{u(x): x \in B_{a \rho}\left(x_{0}\right)\right\},
$$

where $\rho=\left|x_{0}\right| / 2, B_{a p}\left(x_{0}\right)$ is the open ball of center $x_{0}$ and radius $a \rho$, and $C$ depends on $n, a$, the norm of $V$ in $K_{n}$, but not on $x_{0}$. To do this we rescale the problem and consider the function $v=v_{x_{0}}$ defined by

$$
v(x)=u\left(\rho\left(x+x_{0}\right)\right) \text {. }
$$

$v$ is a solution of $-\Delta v+W(x) v=0$ in $B_{1}$, with $W(x)=\rho^{2} V\left(\rho\left(x+x_{0}\right)\right)$.

By Theorem 3.10 of [AS] we get

$$
\sup _{B_{a}} v(x) \leq C \inf _{B_{a}} v(x) .
$$

Moreover, (cf. Theorems 1.2, 1.6, 2.4, 3.7, 3.8 and Proposition 2.2 of [AS]) $C$ may be taken as $C=K /(1-\beta)$, where $K=K(n, a)$ and $\beta$ is any number less than 1 such that

$$
\beta \geq K_{1}(n) \cdot \sup _{x \in B_{1}} \int_{|x-y| \leq 2 a}|x-y|^{2-n}|W(y)| d y .
$$

This choice of $\beta$ is possible for $a$ small enough since

$$
\begin{aligned}
& \sup _{x \in B_{1}} \int_{|x-y| \leq 2 a}|x-y|^{2-n}|W(y)| d y \\
& \quad=\sup _{x \in B_{\rho}\left(x_{0}\right)} \int_{|x-y| \leq 2 a \rho}|x-y|^{2-n} V(y) \mid d y \\
& \quad \leq \sup _{x \in B_{3 R / 2}} \int_{|x-y| \leq a R / 2}|x-y|^{2-n}|V(y)| d y
\end{aligned}
$$


and this integral tends to 0 as $a \rightarrow 0$. This proves (15) and with it (13).

To obtain (12) from (13) we only have to cover the sphere $|x|=\left|x_{0}\right|$ with a finite number of balls $B_{a \rho}\left(x_{i}\right)$ with $\left|x_{i}\right|=\left|x_{0}\right|$. Their number depends only on $a$.

The second step begins by writing $L u=0$ in the form

$$
\Delta u=V^{+} \cdot u-V^{-} \cdot u
$$

where $V^{+}$and $V^{-}$are the positive and negative parts of $V$, i.e. $V^{+}$ $=\frac{1}{2}(V+|V|), V^{-}=\frac{1}{2}(-V+|V|)$. Since $V^{-} \cdot u \geq 0$ we have $\Delta u \leq V^{+} \cdot u$ and we are reduced to study the behaviour at 0 of nonnegative supersolutions of $L u=0$ with a nonnegative potential in $K_{n}$. This is done in the following result, which may have an independent interest and is therefore labeled as

Theorem 4. Let $V$ be a nonnegative potential in $K_{n}\left(B_{R}\right)$ and let $u \in L_{\mathrm{loc}}^{1}\left(B_{R}^{*}\right)$ satisfy: $u \geq 0, \Delta u \in L_{\mathrm{loc}}^{1}\left(B_{R}^{*}\right), V \cdot u \in L_{\mathrm{loc}}^{1}\left(B_{R}^{*}\right)$ and

$$
\Delta u \leq V \cdot u+f \quad \text { in } B_{R}^{*}
$$

for some $f \in L_{\text {loc }}^{1}\left(B_{R}\right)$. If either $V$ is radially symmetric or $u$ satisfies (12), then $u$ is a solution in $B_{R}$ of the equation

$$
-\Delta u+V u=\alpha \delta+\varphi
$$

for some $\alpha \in[0, \infty)$ and $\varphi \in L_{\mathrm{loc}}^{1}\left(B_{R}\right)$. In particular $u, V u \in L_{\mathrm{loc}}^{1}\left(B_{R}\right)$ and

$$
r^{n-2} \bar{u}(r) \rightarrow \alpha / c_{n}, \quad r^{n-1} \bar{u}_{r}(r) \rightarrow-\alpha / s_{n} .
$$

This theorem extends a result by Brezis and Lions [BL], which consider the case $V=$ constant. As in Theorem 1, it happens that $V \in K_{n}$ is the necessary and sufficient condition for the result to hold in the class of nonnegative and radially symmetric potentials.

Proof of Theorem 4. The proof of (18) in [BL] begins by obtaining that $V u \in$ $L_{\text {loc }}^{1}\left(B_{R}\right)$ and $\bar{u}(r)=O\left(r^{2-n}\right)$ as $r \rightarrow 0$. Then it is concluded that the function $\varphi$ defined a.e. in $B_{R}$ as $\varphi(x)=-\Delta u(x)$ belongs to $L_{\text {loc }}^{1}\left(B_{R}\right)$. Finally they prove that the distribution $T=\Delta u+\varphi$ must be a Dirac mass at 0 .

Our proof consists in establishing $V u \in L_{\text {loc }}^{1}\left(B_{R}\right)$. We then write (17) as

$$
\Delta u \leq g \equiv V u+f \in L_{\text {loc }}^{1}\left(B_{R}\right)
$$

and apply [BL] with $V \equiv 0$ to get the conclusion. We proceed as follows. Averaging (17) on the sets $|x|=r$ for $0<r<R / 2$, we get

$$
-\left(r^{n-1} \bar{u}_{r}\right)_{r} \leq r^{n-1}(\overline{V u}(r)+\bar{f}(r)) \text {. }
$$

It is at this stage that we need an extra assumption on $V$ or $u$ in order to relate $\overline{V u}$ to $\bar{V} \cdot \bar{u}$. If $V$ is radially symmetric we get $\overline{V u}=\bar{V} \cdot \bar{u}$; on the other hand if (12) holds then $\overline{V u} \leq c \bar{V} \cdot \bar{u}$. Since $c>1$ we get after substituting into (20)

$$
-\left(r^{n-1} \bar{u}_{r}\right)_{r} \leq c r^{n-1} \bar{V}(r) \bar{u}(r)+r^{n-1} \bar{f}(r) .
$$


Now we remark that $V \in K_{n}$ implies $\bar{V} \in K_{n}$, i.e. (cf. (6)) $\int_{0}^{R / 2} \bar{V}(r) r d r<\infty$. On the other hand $\bar{u} \in C^{1}(0, R)$. Take $0<R_{1}<R / 2$ and integrate (21) over $\left(r, R_{1}\right)$ to obtain

$$
r^{n-1} \bar{u}_{r}(r) \leq c \psi(r)+C, \quad 0<r<R_{1},
$$

where $\psi$ is defined by $\psi(r)=\int_{r}^{R_{1}} \bar{V}(r) \bar{u}(r) r^{n-1} d r$ and $C$ denotes a constant that depends on $R_{1}$ but not on $r$. Dividing (22) by $r^{n-1}$ and integrating again we have

$$
\bar{u}(r) \leq c \int_{r}^{R_{1}} \psi(s) s^{1-n} d s+C r^{2-n}+C .
$$

Now multiply by $r^{n-1} \bar{V}(r)$, integrate once again and use the fact that $\psi$ is nonincreasing to arrive at

$$
\psi(r) \leq\left(\frac{c}{n-2} \psi(r)+C\right) \int_{r}^{R_{1}} s \bar{V}(s) d s, \quad 0<r<R_{1} .
$$

Since $r \bar{V}(r) \in L^{1}(0, R / 2)$, if we choose $R_{1}$ small enough we conclude that $\psi$ is bounded in $\left(0, R_{1}\right)$. This implies that $V u \in L_{\text {loc }}^{1}\left(B_{R}\right)$ since $\overline{V u} \leq c \overline{V u}$ for $0<r<R / 2$. From (23) it follows then that $\bar{u}(r)=O\left(r^{2-n}\right)$ as $r \rightarrow 0$. As explained above, we obtain in this way (18).

Estimate (19) is easy to obtain from (18) by Gauss' theorem. Indeed for $0<r<R$

$$
\begin{aligned}
s_{n} r^{n-1} \bar{u}_{r}(r) & =\int_{|x|=r} \frac{\partial u}{\partial r}(r, \sigma) r^{n-1} d \sigma=\int_{|x|<r} \Delta u d x \\
& =-\alpha+\int_{|x|<r}(V u-\varphi) d x \rightarrow-\alpha \quad \text { as } r \rightarrow 0 .
\end{aligned}
$$

One of the good points of Theorem 4 is that the inequality in (17) allows to replace a potential $V$ by any upper bound. In particular, the negative part of $V$ plays no role in the result. This has for instance the following consequence.

Proposition 5. Let $V$ be a radially symmetric potential such that $V^{+} \in K_{n}$ but $V^{-} \notin K_{n}$ and let $u$ be a nonnegative solution of (1) in $B_{R}^{*}$ with $-\Delta u+$ $V u \in L_{\mathrm{loc}}^{1}\left(B_{R}\right)$. Then the singularity at 0 is removable. (In other words, all singularities are of oscillatory type.)

Proof. We apply Theorem 4 with potential $V^{+}$to conclude that $\bar{u}(r) \approx$ $\left(\alpha / c_{n}\right) r^{2-n}$ near the origin and $u, \Delta u-\alpha \delta$ and $V^{+} u \in L_{\mathrm{loc}}^{1}\left(B_{R}\right)$. On the other hand, if $\alpha>0$ we get

$$
\begin{aligned}
\int_{|x|<R / 2} V^{-}(x) u(x) d x & =C \int_{0}^{R / 2} V^{-}(r) \bar{u}(r) r^{n-1} d r \\
& \geq C \int_{0}^{R / 2} r V^{-}(r) d r=\infty .
\end{aligned}
$$


Since $V^{-} \cdot u=V^{+} u-\Delta u$ we arrive at a contradiction. Hence $\alpha=0$ and the singularity is removable.

The last step in the proof of Theorem 1 starts from the application of Theorem 4 to $\Delta u \leq V^{+} \cdot u$. By (12) and (19) we have

$$
u(x) \leq C \alpha|x|^{2-n}
$$

if $|x|$ is small. Here in the sequel $C$ will denote different positive constants depending on $n, u, V$ and $R$ but not on $|x|$. Since $V \in K_{n}$ (25) implies that $V u \in L_{\text {loc }}^{1}\left(B_{R}\right)$. Now the function $\varphi$ in (18) equals $V^{-} \cdot u$ a.e. in $B_{R}$, therefore we have

$$
-\Delta u+V u=\alpha \delta \quad \text { in } D^{\prime}\left(B_{R}\right)
$$

and the proof ends once we establish the asymptotic behaviour (8). For that we let $v$ be the solution of the problem

$$
-\Delta v=\alpha \delta \text { in } D^{\prime}\left(B_{R / 2}\right), \quad v=u \quad \text { on }|x|=R / 2 .
$$

It is clear that $v \in C\left(B_{R / 2}^{*}\right)$ and $v(x)|x|^{n-2} \rightarrow \alpha / c_{n}$ as $|x| \rightarrow 0$. Therefore, we have to prove that $w \equiv u-v$ is $o\left(|x|^{2-n}\right)$ as $|x| \rightarrow 0$. Notice that $w$ solves

$$
\Delta w=V u \text { in } D^{\prime}\left(B_{R / 2}\right), \quad w=0 \quad \text { on }|x|=R / 2,
$$

hence it can be represented in the form

$$
w(x)=-\int_{B_{R / 2}} G(x, y) V(y) u(y) d y,
$$

where $G$ is the Green function for the Laplace operator $-\Delta$ in $B_{R / 2}$. It is well known that $G(x, y)$ is a $C^{\infty}$-function for $x, y \in \bar{B}_{R / 2}, x \neq y$. Moreover

$$
0 \leq G(x, y) \leq c_{n}|x-y|^{2-n}
$$

and $G$ is positive for $x, y \in B_{R / 2}$. We shall estimate $w(x)$ for $|x|$ small using all these data. In fact, let $R_{1}=\min \left(4, R / 2, R^{2} / 4\right)$. For $|x| \leq R_{1}$ we have

$$
\begin{aligned}
w(x) \leq & c_{n} \int_{|y| \leq R / 2}|x-y|^{2-n}|V(y)| u(y) d y \\
\leq & \int_{|y| \leq|x| / 2}|x-y|^{2-n}|V(y)| u(y) d y \\
& +\int_{|x| / 2<|y| \leq|x|^{1 / 2}}|x-y|^{2-n}|V(y)| u(y) d y \\
& +\int_{|x|^{1 / 2}<|y| \leq R / 2}|x-y|^{2-n}|V(y)| u(y) d y \\
= & I_{1}+I_{2}+I_{3} .
\end{aligned}
$$


A bound for $I_{1}$ for $|x| \approx 0$ is obtained as follows. Since $|y| \leq|x| / 2$, we have $|x-y|^{2-n} \leq 2^{n-2}|x|^{2-n}$, so that

$$
\begin{aligned}
I_{1} & \leq 2^{n-2}|x|^{2-n} \int_{|y| \leq|x| / 2}|V(y)| u(y) d y \\
& =C|x|^{2-n} \int_{|y| \leq|x| / 2}|y|^{2-n} V(y) d y=o\left(|x|^{2-n}\right)
\end{aligned}
$$

since $V \in K_{n}$. Likewise

$$
I_{3} \leq\left(|x|^{1 / 2}-|x|\right)^{2-n} \int_{|y| \leq R} u(y)|V(y)| d y=O\left(|x|^{1-n / 2}\right) .
$$

Finally let $D=\left\{y \in B_{R}:|x| / 2 \leq|y| \leq|x|^{1 / 2}\right\}$. We have

$$
\begin{gathered}
I_{2} \leq C \int_{D}|x-y|^{2-n}|y|^{2-n}|V(y)| d y \\
C|x|^{2-n} \int_{D}|x-y|^{2-n}|V(y)| d y .
\end{gathered}
$$

Since $V \in K_{n}$ the last integral vanishes as $|x| \rightarrow 0$, hence $I_{2}=o\left(|x|^{2-n}\right)$. We conclude that $w=o\left(|x|^{2-n}\right)$, which ends the proof of Theorem 1 .

\section{Proof of Theorem 2}

The proof relies on some properties of the Green function for the operator $L=-\Delta+V$ in a small enough ball $B_{R}$. In [CFZ] (see also [CFZ2]) Cranston, Fabes and Zhao study the conditional gauge $F$ associated to the diffusion process generated by $L$ in a bounded Lipschitz domain $D$ of $\mathbf{R}^{n}$ and its relation with the Green function, $G_{L}$, of $L$ in $D$. We take the following results from [CFZ].

(a) Either $F$ is infinite everywhere or there exist positive constants $c_{1}, c_{2}$ such that

$$
0<c_{1} \leq F(X, Y) \leq c_{2}
$$

for all $x, y \in D$ (cf. Theorem 2.2).

(b) In the latter case $F$ is continuous in $\bar{D} \times \bar{D}$ (Theorem 4.9) and

$$
G_{L}(x, y)=F(x, y) G(x, y)
$$

in $D * D, G$ being the Green function for $-\Delta$ (Theorem 4.7).

(c) $F$ is finite if and only if $\inf \left(\operatorname{spec}\left(\left.L\right|_{D}\right)\right)>0$.

Now, the infimum of the spectrum of $L$ in $D$ can be made positive by choosing $D$ to be a sufficiently small ball. This is a consequence of the following embedding result for Kato potentials proved by Fabes [F], cf. also [CFG].

Lemma 6. For every $\varepsilon>0$ there exists $r>0$ such that

$$
\int u^{2}|V| d x \leq \varepsilon \int|\nabla u|^{2}
$$

for every $u \in H_{0}^{1}\left(B_{r}\right)$. 
In the sequel we fix $D=B_{r}$ with such an $r$. We also put $U(x)=G_{L}(x, 0)$. Then $U \in C\left(B_{r}^{*}\right), U$ vanishes for $|x|=r, V U \in L^{1}\left(B_{r}\right)$ and (7), (8) hold with $\alpha=1$.

Assume that $u$ is a function as in Theorem 2 and let $f=\Delta u-V u \in L^{p}\left(B_{r}\right)$. We define

$$
u_{1}(x)=\int_{B_{r}} G_{L}(x, y) f(y) d y .
$$

It is clear that $u_{1} \in C\left(B_{r}\right)$ and $L u_{1}=f$. Put $v=u-u_{1}$. It satisfies $L v=0$ in $B_{r}^{*}$ and

$$
v(x) \geq-c|x|^{2-n}
$$

for some constant $c$ and $|x| \leq r^{\prime}<r$. Therefore the function $w=v+\left(c / c_{n}\right) U$ is a nonnegative solution of $L u=0$ in a small punctured neighbourhood of 0 . By Theorem 1 there exists $\alpha_{1} \geq 0$ such that

$$
L w=\alpha_{1} \delta \text { in } D^{\prime}\left(B_{r^{\prime}}\right)
$$

and

$$
w(x)|x|^{n-2} \rightarrow \alpha_{1} / c_{n} \text { as }|x| \rightarrow 0 .
$$

Put now $\alpha=\alpha_{1}-\left(c / c_{n}\right)$ and let $g=u-\alpha U=w-\alpha_{1} U+u_{1}$. The proof will be complete if we show that $g$ is continuous for $x \approx 0$. For this observe that

$$
L\left(g-u_{1}\right)=L\left(w-\alpha_{1} U\right)=0 \quad \text { in } D^{\prime}\left(B_{r^{\prime}}\right)
$$

and $g-u_{1}=o\left(|x|^{2-n}\right)$ as $|x| \rightarrow 0$, hence $g-u_{1} \in L^{1}\left(B_{r^{\prime}}\right)$. In this situation Theorem 15 of [AS] implies that $g-u_{1}$ is a continuous function in $B_{r^{\prime}}$ and we are done.

Acknowledgements. The authors are grateful to Gene Fabes for valuable information. The second author would like to thank the Universidad Autónoma for its hospitality during her stay in Madrid to work on this project.

\section{REFERENCES}

[AS] M. Aizenman and B. Simon, Brownian motion and Harnack inequality for Schrödinger operators, Comm. Pure Appl. Math. 35 (1982), 209-273.

[BL] H. Brezis and P. L. Lions, A note on isolated singularities for linear elliptic equations, Mathematical Analysis and Applications, (A. L. Nachbin, ed.), Academic Press, New York, 1981, pp. 263-266.

[CFG] F. Chiarenza, E. Fabes and N. Garofalo, Harnack's inequality for Schrödinger operators and the continuity of solutions, Proc. Amer. Math. Soc. 98 (1986), 415-425.

[CFZ] M. Cranston, E. Fabes and Z. Zhao, Conditional gauge and potential theory for the Schrödinger equation, Trans. Amer. Math. Soc. 307 (1988), 171-194.

[CFZ2] _ , Potential theory for the Schrödinger equation, Bull. Amer. Math. Soc. 15 (1986), 213216.

[F] E. Fabes, Private communication. 
[GS] D. Gilbarg and J. Serrin, On isolated singularities of solutions of second-order elliptic differential equations, J. Analyse Math. 4 (1955-56), 309-340.

[K] T. Kato, Schrödinger operators with singular potentials, Israel J. Math. 13 (1973), 135-148.

[S1] J. Serrin, Local behaviour of solutions of quasi-linear equations, Acta Math. 111 (1964), 247302.

[S2] __ Isolated singularities of solutions of elliptic equations, Acta Math. 113 (1965), 219-240.

[Si] B. Simon, Schrödinger semigroups, Bull. Amer. Math. Soc. 7 (1982), 447-526.

[SW] J. Serrin and H. Weinberger, Isolated singularities of solutions of linear equations, Amer. J. Math. 88 (1966), 258-272.

[VY1] J. L. Vazquez and C. Yarur, Singularités isolées et comportement à l'infini des solutions de l'équation de Schrödinger stationnaire, C.R. Acad. Sci. Paris I 300 (1985), 105-108.

[VY2] __, Isolated singularities of the solutions of the Schrödinger equation with a radial potential, Arch. Rational Mech. Anal. 98 (1987), 251-284.

Departamento de Matemáticas, Universidad Autónoma de Madrid, 28049 Madrid, SPAIN

Departamento de Matemática y Ciencias de la Computación, Universidad de Santiago de Chile, Casilla 5659, Correo 2, Santiago, Chile 